\title{
Deep Optimized Electrodes and Frequency Bands in the Phase Space for Identification of Seizures
}

This paper was downloaded from TechRxiv (https://www.techrxiv.org).

\section{LICENSE}

CC BY 4.0

SUBMISSION DATE / POSTED DATE

$22-12-2021 / 23-12-2021$

\section{CITATION}

Rukhsar, Salim; Panda, Samhita; Tiwari, Anil Kumar (2021): Deep Optimized Electrodes and Frequency Bands in the Phase Space for Identification of Seizures. TechRxiv. Preprint. https://doi.org/10.36227/techrxiv.17372024.v1

$\mathrm{DOI}$ 


\title{
Deep Optimized Electrodes and Frequency Bands in the Phase Space for Identification of Seizures
}

\author{
Salim Rukhsar, Anil Kumar Tiwari, and Samhita Panda
}

\begin{abstract}
The electroencephalogram (EEG) has been extensively used to record brain signals for the analysis of neurological diseases over several decades. Epilepsy is the one of the most common neurological disorder in the world. It is associated with seizures, which are a clinical manifestation of abnormal, excessive excitation and synchronisation of a population of cortical neurons, resulting in abrupt changes in consciousness, movement, behaviour, and feelings. Our goal in this letter is to propose a computationally efficient EEG based system that can detect seizures with improved detection performance. To deeply optimize the electrodes and frequency bands, we proposed a fast computational algorithm by finding the critical electrodes and optimal sub-bands of EEG signals. We used wavelet packet decomposition (WPD) to find optimal sub-bands and maximum relevance minimum redundancy (mRMR) method for electrodes selection. It is found that the portrait of the features in the phase space depicted promising discriminating ability to detect seizure efficiently. Experimental results show that the proposed method outperformed the state-of-art methods and provided $100 \%$ and $\mathbf{9 4 . 7 9} \%$ of detection accuracy on UBonn and CHB-MIT datasets, respectively, at a significantly reduced computational cost.
\end{abstract}

Index Terms-Epileptic seizure, Wavelet packet decomposition, Phase Space Reconstruction, maximum relevance minimum redundancy

\section{INTRODUCTION}

$\mathbf{E}$ PILEPSY is a chronic neurological disease which is considered to be the one of the most common neurological disorder. Current statistics show that the disease affects over 70 million people worldwide [1]. Epileptic seizures are sudden and uncontrolled electrical disturbances in the brain, directly affecting the central processing that causes various types of problems in movements, behavior, feelings, and consciousness. It can affects men and women's irrespective of their ages, socioeconomic, racial, or geographic circumstances. Additionally, the unpredictable behavior of patients with epilepsy, along with the stress related concerns it poses that renders it difficult to manage.

To improve the quality of life of such patients, it is important to have a convenient system for accurate detection of seizures. The most common method of recording the brain electrical activity is the multi-lead electroencephalogram (EEG). The processing of such signals for manual identification of seizure events by an expert is expensive and time-consuming task. For the last few decades, researchers have been working to develop an automatic EEG-based epilepsy detection system.

Salim Rukhsar and Anil Kumar Tiwari are with the Department of Electrical Engineering, Indian Institute of Technology Jodhpur, Rajasthan, INDIA, 342037, India (e-mails: \{rukhsar.1, akt\} @iitj.ac.in)

Samhita Panda is with the Department of Neurology, All India Institute of Medical Sciences, Jodhpur, Rajasthan, INDIA (email:samhitapanda@yahoo.com)
It is needed for quick diagnosis as it can replace the timeconsuming and exhausting human visual assessment process with minimal error [2] [3]. To analyse EEG signals for seizure identification, a variety of feature extraction approaches have been reported [4] [5]. These approaches primarily analyse the EEG signal in the time and frequency domains, assuming that their characteristic is stationary [1] [6]. The non-stationary characteristics of such signals led to the requirement of the signal analysis in the non-linear dynamics (chaos) and timefrequency domain [7]. As a result, some studies have been proposed that extracted nonlinear features of EEG signals for epilepsy diagnosis such as the recurrent plot, Lyapunov exponent, and approximate entropy (ApEn) [8] [9].

Various supervised and unsupervised machine learning algorithms are proposed for the above stated classification. Due to minimization of structural risk and generalization ability, support vector machine (SVM) methods have been widely used for the classification of handcrafted features in the recent years [4] [10]. Yavuz et.al. [11] took the advantage of the artificial neural networks (ANNs) to identify epileptic seizures efficiently. The K-nearest Neighbourhood (KNN) method is also extensively used to identify seizures [5] [12]. The employment of a one-dimensional $\mathrm{CNN}$ in conjunction with a bidirectional long short-term memory (LSTM) improved accuracy, according to Abdelhameed et al. [13]. Recent research has attempted to use deep learning (DL) to reduce the time and effort required in hand-engineered features, as well as to identify unique characteristics to improve the performance [14].

The remaining sections of this letter are organised as follows: Section II covers the proposed methods, including the dataset used and the classification methods such as mRMR, WPD, phase space reconstruction, and LSSVM. The experimental results are given in the section III. Finally, Section IV has the conclusion of this work and future research directions.

\section{Methodology}

The goal of the proposed research is to develop an epileptic seizure detection system that uses a minimal number of channels, and frequency sub-bands containing seizure signature are identified for two and three classes of signal. The maximal relevance minimal redundancy (mRMR) minimizes computational complexity and the use of wavelet packet decomposition (WPD) leads to discover the frequency sub-bands. Frequency sub-bands in the phase space portrayed exhibited excellent accuracy by using support vector machine (SVM) for classification. 


\section{A. Dataset}

Two freely available datasets are used to assess the effectiveness of the proposed study in this letter. The one of the datasets is from the university of Bonn (Germany) [21]. This dataset is diveded into five categories labelled A through $\mathrm{E}$. The $\mathrm{A}$ and B sets represent normal state signals of five healthy individuals who have their eyes open and closed, respectively. The $\mathrm{C}$ and $\mathrm{D}$ sets were recorded during the interictal condition. Set C consists of hippocampal formation of the opposite hemisphere of the brain while set D consists of epileptogenic zones. The last set E contains the data from seizure cases. Each dataset has 100 segments of 23.61 seconds with sampling rate of 173.61 $\mathrm{Hz}$ that correspond to a single scalp EEG electrode.

The second dataset was obtained from the Children's Hospital of Boston-Massachusetts Institute of Technology (CHBMIT) and is also freely accessible on https:/PhysioNet.org/ [22]. It contains 198 seizures acquired in the period of approximately 987 hours. This scalp EEG data is of 24 patients (17 females, 5 males), aged between 1.5 and 22 years old. The sampling rate of the recording is $256 \mathrm{~Hz}$ at the sample resolution of 16-bit over 23 channels using bipolar montage.

\section{B. Channel selection}

It is vital to decrease computational expenses in order to have a fast and low-memory system. In this work, we use a mutual information-based filter selection technique called mRMR [15] as it can effectively reduce redundant features retaining the relevant ones. This technique entails finding a feature subset within which each selected feature has the highest mutual information (MI) with the target class and the lowest average MI among themselves. In practice, a method of incremental search is used to maximise the given condition:

$$
\max _{X_{j} \in S_{n} / X}\left(I\left(X_{j}, T\right)-\frac{1}{n-j+1} \sum_{x_{i} \in X} I\left(X_{j}, X_{i}\right)\right)
$$

where $T$ is the target class, $S_{n}=X_{1}, X_{2}, \cdots, X_{n}$ is the entire $n$ feature set, $X$ is the selected feature set, and $I$ is the mutual information defined as

$$
I(X, Y)=\sum_{y \in \Omega_{Y}} \sum_{x \in \Omega_{x}} p(x, y) \log \left(\frac{p(x, y)}{p(x) p(y)}\right)
$$

Where $p(x), p(y)$ and $p(x, y)$ are PMF and Joint PMF respectively. mRMR iteratively maximizes mutual information between a feature $X_{j} \in S_{n}$ and the target class $T$, while minimizes MI between new feature $X_{j}$ and previously chosen feature $X_{i} \in X$. In this study, we selected only seven channels out of twenty three and only four frequency sub-bands out of eight.

\section{Wavelet Packet Decomposition}

The Wavelet Packet Decomposition (WPD) is an extension of discrete wavelet transform. The Wavelet transform decomposes only low frequency components (approximation), while keeping the high frequency components (detail) as it is. This issue is resolved by decomposing detailed components successively using WPD [16]. The wavelet packet, $\psi_{j, k}^{i}$ is described below, where $i, j$ and $k$ are the modulation, dilation, and translation parameters.

$$
\psi_{j, k}^{i}(t)=2^{-j / 2} \psi^{i}\left(2^{-j} t-k\right)
$$

Here $i=1,2 \ldots j^{n}$ and $n$ is the level of decomposition. The wavelet $\psi^{i}$ can be found by the recursive relationship described below:

$$
\begin{aligned}
\psi^{2 i}(t) & =\frac{1}{\sqrt{2}} \sum_{k=-\infty}^{\infty} h(k) \psi^{i}\left(\frac{t}{2}-k\right) \\
\psi^{2 i+1}(t) & =\frac{1}{\sqrt{2}} \sum_{k=-\infty}^{\infty} g(k) \psi^{i}\left(\frac{t}{2}-k\right)
\end{aligned}
$$

here $\psi^{1}(t)$ is the mother wavelet, $h(k)$ and $g(k)$ are quadrature mirror discrete filters with the scaling and the mother wavelet function.

\section{Phase Space Reconstruction}

The phase space reconstruction (PSR) method is particularly useful for unfolding nonlinear dynamics of a signal. Due to the non-stationary nature of EEG signal, the portrayal of the signal in the phase space provides the visual representation of the signal dynamics that has evolved over time [17]. Since the EEG signal characteristics changes from chaotic to synchronized during the seizure, the portrait of the signal in PSR provides better discriminability. Let the set of m-data point of EEG signal be represented as $A=\left\{a_{1}, a_{2}, \ldots a_{m}\right\}$. In this method, delayed dimension $A_{r}$ is reconstructed from $\mathrm{A}$ as $A_{r}=\left[\begin{array}{llll}a_{r} & a_{r+1} & a_{r+2} \ldots \ldots & a_{m-(p-1) \tau+(r-1)}\end{array}\right]^{T}$ with $r=$ $1,1+\tau, 1+2 \tau, \ldots ., 1+p \tau$, where $\tau$ is the time lag/delay, and $p$ is the embedding dimension of PSR, described below. The $p$-dimensional data in PSR can be represented as $Z=\left[\begin{array}{llll}A_{1} & A_{1+\tau} & A_{1+2 \tau} \ldots \ldots \ldots . & A_{1+(p-1) \tau}\end{array}\right], Z \in \mathbb{R}^{p}$.

The optimal time delay $\tau$ to unfold the dynamics of signal can be found by using average mutual information (AMI) due to its natural ability for non-linear time series data [18]. To evaluate the delay time $\tau$, following equation is used for AMI computation:

$$
I(\tau)=\sum_{k=1}^{p-1} P\left(A_{1}, A_{1+k \tau}\right) \cdot \log \left(\frac{P\left(A_{1}, A_{1+k \tau}\right)}{P\left(A_{1}\right) \cdot P\left(A_{1+k \tau}\right)}\right)
$$

where $P\left(A_{1}\right)$ and $P\left(A_{1}, A_{1+k \tau}\right)$ are the PMF of $A_{1}$ and joint PMF of $A_{1}$ and $A_{1+k \tau}$, respectively. $I(\tau)$ shows the statistical dependence of the reconstructed data. The suitable value of $\tau$ is the first local minima, as $I(\tau)$ decreases non-monotonously. The false nearest neighbor (FNN) approach has been used to compute the optimal embedding dimension $p$. The FNN algorithm searches for the minimal dimension that unfolds the dynamics of one dimensional data. False neighbours are those who are no longer neighbours of a data point in PSR when it moves from $p$ to $p+1$ dimension. The square of euclidean distance between $a_{i}$ and its neighbor can be computed as follows:

$$
R_{p}^{2}(i, h)=\sum_{k=1}^{p-1}\left[a_{i+k \tau}-a_{i+k \tau}^{(h)}\right]^{2}
$$




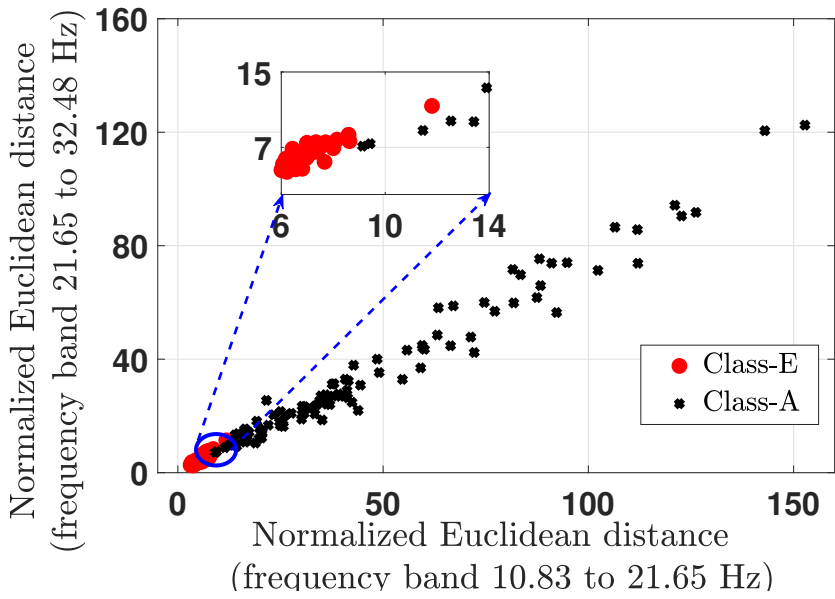

(a)

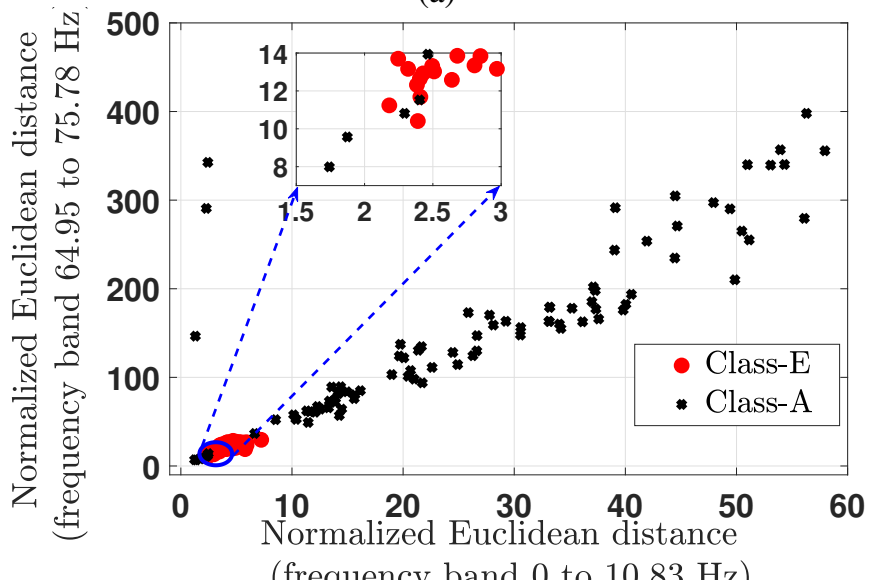

(b)

Fig. 1. Sub-bands feature (Normalized Euclidean distance in PSR) discriminability between class A (interictal) and class $\mathrm{E}$ (ictal) in different frequency bands (a) between the bands 10.83 to $21.65 \mathrm{~Hz}$, and 21.65 to $32.48 \mathrm{~Hz}$ and (b) between the bands 0 to 10.83 and 64.95 to $75.78 \mathrm{~Hz}$

where $i=1,2,3, \cdots, m-(p-1) \tau$. Here $h=1$ is considered for the closest neighbor of data point $a_{i+k \tau}$, consequently $h$ is dropped from $R_{p}^{2}(i, h)$. The following two criteria have to jointly fail to be consider a point as nearest neighbors: $\sqrt{\left(\frac{R_{p+1}^{2}(i)-R_{p}^{2}(i)}{R_{p+1}^{2}(i)}\right)}>R_{t o l}$, and $\frac{R_{p+1}(i)}{R_{A}}>A_{t o l}$. We used $A_{t o l}=2$ and $R_{t o l}=15$ as recommended, and $R_{A}$ is the standard deviation of data A [19]. The sum of FNN of entire $i$ for every $p$ is calculated until the proportion of FNN tends to zero or very small and continue to follow the same.

The phase space is represented for each decomposed frequency bands obtained using WPD. We used euclidean distance as feature due to synchronization of brain waves during the seizure. The below expression of euclidean distance is used to compute for one epoch.

$$
x_{j, k}=\sum_{j=1}^{L} \sqrt{\sum_{k=1}^{p}\left(a_{j+(k-1) \tau}-a_{j+k \tau}\right)^{2}}
$$

Here $\mathrm{L}=1024$ length of the $4 \mathrm{sec}$ epoch for CHB-MIT dataset.
TABLE I

Performance of the Proposed METHod on the Bonn Dataset

\begin{tabular}{|l|l|l|l|l|l|}
\hline $\begin{array}{l}\text { Classification } \\
\text { Class }\end{array}$ & $\begin{array}{l}\text { Accuracy } \\
(\%)\end{array}$ & $\begin{array}{l}\text { Specificity } \\
(\%)\end{array}$ & $\begin{array}{l}\text { Sensitivity } \\
(\%)\end{array}$ & $\begin{array}{l}\text { Precision } \\
(\%)\end{array}$ & $\begin{array}{l}\text { F1 } \\
\text { Score }\end{array}$ \\
\hline $\mathrm{A} / \mathrm{E}$ & 100 & 100 & 100 & 100 & 1 \\
\hline $\mathrm{C} / \mathrm{E}$ & 99.00 & 98.50 & 99.00 & 98.41 & 0.987 \\
\hline $\mathrm{A} / \mathrm{C} / \mathrm{E}$ & 98.50 & 98.47 & 99.14 & 98.05 & 0.986 \\
\hline
\end{tabular}

\section{E. Least Square Support Vector Machine}

To classify the ictal and interictal features effectively, we used least squares support vector machine (LSSVM) due to kernel trick to map the non-linear separable dataset into a higher dimensional space. For binary class problem in SVM, we use the below decision function [20]:

$$
g(x)=\operatorname{sign}\left[w^{T} \phi(x)+b\right]
$$

where $w$ is $d$-dimensional weight vector, $\phi(x)$ is the mapping function that maps $x$ into the $d$-dimensional space, and $b$ is the bias. To find the parameters $w$ and $b$, the optimization problem is described as follows:

$$
\operatorname{minimize}(w, b, e)=\frac{1}{2} w^{T} w+\frac{\gamma}{2} \sum_{i=1}^{M} e_{i}^{2}
$$

subject to equality constraints $y_{i}\left[w^{T} \phi\left(x_{i}\right)+b\right]=1-e_{i}, i=$ $1,2, \ldots, M$. Where $e_{i}^{2} \in \mathbb{R}$ are error variables and $\gamma \geq 0$ is a regularization constant. Here $\left\{x_{i}, y_{i}\right\}_{i=1}^{M}$ are the $\mathrm{M}$ training input-output pairs, $y_{i}=1$ if $x_{i}$ belongs to class-I and -1 if $x_{i}$ belongs to class-II respectively. The Lagrangian's multipliers $\beta_{i}$ can be expressed for (11) as follows:

$$
L(w, b, e ; \beta)=J(w, b, e)-\sum_{i=1}^{M} \beta_{i}\left(y_{i}\left[w^{T} \phi\left(x_{i}\right)+b\right]+e_{i}-1\right)
$$

Where $\beta=\left[\beta_{1}, \beta_{2} \ldots \beta_{N}\right]^{T}$. Solving (12), Least square SVM classifier is obtained as follows:

$$
f(x)=\sum_{i=1}^{M} \beta_{i} y_{i} K\left(x, x_{i}\right)+b
$$

Where $K\left(x, x_{i}\right)$ is a kernel function. In this work, we used Radial Basis Function defined as follows:

$$
K\left(x, x_{i}\right)=\exp \left(-\frac{\left\|x-x_{i}\right\|}{2 \sigma^{2}}\right)
$$

Where $\sigma$ is width controlling parameters of the RBF function.

\section{EXPERIMENT}

In this study, maximum relevance minimum redundancy (mRMR) is used to choose the most important channels for the CHB-MIT dataset and the most suitable frequency sub-bands for UBonn dataset. For decomposition of the raw EEG signal, wavelet packet decomposition is used. Owing to its simple and computationally efficient approach for analysis, Haar wavelet is used as mother wavelet and the decomposition is done up to the three levels. Responsible sub-bands for seizure detection 
TABLE II

Performance of the Proposed Method on the CHB-MIT Dataset

\begin{tabular}{|l|l|l|l|l|l|l|l|}
\hline $\begin{array}{c}\text { Patients } \\
\text { Id }\end{array}$ & $\begin{array}{l}\text { No of } \\
\text { Seizures }\end{array}$ & $\begin{array}{l}\text { Seizure } \\
\text { interval } \\
(\mathrm{sec})\end{array}$ & $\begin{array}{l}\text { Acc } \\
(\%)\end{array}$ & $\begin{array}{l}\text { Spe } \\
(\%)\end{array}$ & $\begin{array}{l}\text { Sen } \\
(\%)\end{array}$ & $\begin{array}{l}\text { Pre } \\
(\%)\end{array}$ & $\begin{array}{l}\text { F1- } \\
\text { Score }\end{array}$ \\
\hline chb01 & 7 & 442 & 98.61 & 98.61 & 98.61 & 98.61 & 0.986 \\
chb02 & 3 & 172 & 98.67 & 98.67 & 98.67 & 98.67 & 0.987 \\
chb03 & 7 & 402 & 99.64 & 99.57 & 99.71 & 99.71 & 0.996 \\
chb04 & 4 & 378 & 94.85 & 95.26 & 94.45 & 94.40 & 0.948 \\
chb05 & 5 & 558 & 99.05 & 100.0 & 99.03 & 99.02 & 0.995 \\
chb06 & 10 & 153 & 85.29 & 87.50 & 83.33 & 82.35 & 0.848 \\
chb07 & 3 & 325 & 95.39 & 95.70 & 95.08 & 95.05 & 0.953 \\
chb08 & 5 & 919 & 95.55 & 94.69 & 96.45 & 96.52 & 0.956 \\
chb09 & 4 & 276 & 99.06 & 98.56 & 99.58 & 99.58 & 0.990 \\
chb10 & 7 & 347 & 98.27 & 97.97 & 98.58 & 98.59 & 0.983 \\
chb11 & 3 & 806 & 97.19 & 96.50 & 97.91 & 97.94 & 0.972 \\
chb12 & 40 & 1475 & 87.88 & 90.23 & 85.71 & 84.85 & 0.875 \\
chb13 & 12 & 535 & 91.05 & 91.11 & 90.98 & 90.96 & 0.910 \\
chb14 & 8 & 169 & 79.57 & 81.39 & 78.12 & 76.92 & 0.791 \\
chb15 & 20 & 1992 & 92.09 & 92.47 & 91.72 & 91.65 & 0.920 \\
chb16 & 10 & 84 & 96.94 & 96.00 & 97.91 & 97.96 & 0.969 \\
chb17 & 3 & 293 & 99.80 & 99.61 & 100.0 & 100.0 & 0.998 \\
chb18 & 6 & 317 & 93.05 & 89.95 & 96.67 & 96.93 & 0.933 \\
chb19 & 3 & 236 & 97.09 & 96.42 & 97.78 & 97.82 & 0.971 \\
chb20 & 8 & 294 & 92.41 & 89.63 & 95.61 & 95.91 & 0.926 \\
chb21 & 4 & 199 & 90.66 & 93.01 & 88.55 & 87.93 & 0.904 \\
chb22 & 3 & 204 & 99.02 & 98.88 & 99.15 & 99.16 & 0.990 \\
chb23 & 7 & 424 & 99.05 & 99.59 & 98.53 & 98.52 & 0.990 \\
\hline Average & - & - & 94.79 & 94.84 & 94.87 & 94.71 & 0.947 \\
\hline
\end{tabular}

TABLE III

COMPARISON BETWEEN THE PROPOSED SYSTEM AND OTHERS WORKS ON THE SAME DATASET

\begin{tabular}{|c|c|c|c|c|c|}
\hline Ref. & $\begin{array}{l}\text { Task } \\
\text { /Patients }\end{array}$ & Features +classifier & $\begin{array}{l}\text { Acc } \\
(\%)\end{array}$ & $\begin{array}{l}\text { Sen } \\
(\%)\end{array}$ & $\begin{array}{l}\text { Spec } \\
(\%)\end{array}$ \\
\hline$[4]$ & $\mathrm{A} / \mathrm{E}$ & Cepstral analysis + GRNN & 99.5 & 99 & 100 \\
\hline [3] & $\mathrm{A} / \mathrm{E}$ & DCT+H-expo+ARMA+SVM & 94.85 & 95.40 & 94.30 \\
\hline Ours & $\mathrm{A} / \mathrm{E}$ & WPD+mRMR+PSR+LSSVM & 100 & 100 & 100 \\
\hline [6] & $\mathrm{C} / \mathrm{E}$ & Stein kernel-based SR & 98.50 & 99.23 & 98.17 \\
\hline [3] & $\mathrm{C} / \mathrm{E}$ & DCT+H-expo+ARMA+SVM & 97.50 & 98.00 & 97.00 \\
\hline Ours & $\mathrm{C} / \mathrm{E}$ & WPD+mRMR+PSR+LSSVM & 99.00 & 98.50 & 99.00 \\
\hline [10] & $\mathrm{A} / \mathrm{C} / \mathrm{E}$ & FB-series+WMRPE+LSSVM & 97.30 & - & - \\
\hline [7] & $\mathrm{A} / \mathrm{C} / \mathrm{E}$ & $\begin{array}{l}\text { FSWT+ CSoS, FuzzyEn, } \\
\text { HFD, t-SNE + KNN }\end{array}$ & 99.69 & - & - \\
\hline Ours & $\mathrm{A} / \mathrm{C} / \mathrm{E}$ & WPD+mRMR+PSR+LSSVM & 98.50 & 98.50 & 99.00 \\
\hline$[8]$ & $\begin{array}{l}6 \\
\text { patients }\end{array}$ & $\mathrm{BR}+\mathrm{RP}+\mathrm{KNN}$ & 85.00 & 92.30 & 88.20 \\
\hline$[2]$ & $\begin{array}{l}23 \\
\text { patients }\end{array}$ & $\begin{array}{l}\text { Adversarial training+ } \\
\text { features }\end{array}$ & 91.71 & 91.09 & 94.73 \\
\hline Ours & $\begin{array}{l}23 \\
\text { patients }\end{array}$ & WPD+mRMR+PSR+LSSVM & 94.79 & 94.87 & 94.84 \\
\hline
\end{tabular}

are selected by mRMR. The AMI and FNN provided the best time delay and embedding dimensions that enabled extraction of the best possible features. For Bonn data set, 200 epochs are made for each class. 10-fold cross validation is used for classification using LSSVM with the Gaussian kernel. For the binary classification, the LSSVM is trained for 360 epochs and for classification into three classes, classifier is trained for 540 epochs. For the Bonn dataset, mRMR explicitly showed that the responsible sub-bands for the seizure detection are $10.82-21.65 \mathrm{~Hz}, 21.65-32.48 \mathrm{~Hz}, 32.48-43.30 \mathrm{~Hz}$, and $43.30-$ $54.13 \mathrm{~Hz}$. These four bands are used for seizure detection which shows convincing results compared to those reported in the state-of-art methods at a lower computational cost.
Fig. 1 shows the discriminating ability of features in different bands for binary classification. Table I compares the findings for two and three classes, demonstrating that the proposed method outperforms state-of-the-art methods. For the CHBMIT dataset, mRMR is able to find the best possible electrodes for seizure detection. It is found that the seven electrodes out of twenty three are able to provide classification of seizures with equal or greater than $p=0.98$ value compared to the use of all electrodes for classification. Each selected channel is analysed with epoch size of four second $(256 \times 4=1024$ samples). In this way the dimension of data goes to $(7 \times 1024)$ and each epoch is classified as either dominantly seizure or non-seizure (using expert labels). The classification result of each patient is summarized in the Table II.

Table III highlights the comparisons between the proposed method and state-of-the-art methods. The state-of-art study relied on a dedicated feature extraction method and a classifier based on an artificial neural network. Several methods for feature extraction have been used like cepstral analysis, Discrete Cosine Transform (DCT), Stein kernel-based sparse representation and Fourier-Bessel series expansion (FBSE) with weighted multi-scale Renyi permutation entropy (WMRPE). For two class $\mathrm{A} / \mathrm{E}$ and $\mathrm{C} / \mathrm{E}$, the proposed work outperforms the previous work [3] [4] [6] with only four sub band features. It not only shows better results but also establishes the frequency bands to be analysed for seizure detection. For the three class $\mathrm{A} / \mathrm{C} / \mathrm{E}$ task, it is comparably better with many previous works but slightly lagging in performance as compared to the work proposed by Zhang et. al [7]. However, the robustness of our work can be seen because of work on both UBonn and CHB-MIT datasets, as compared to Zhang et. al [7] who only worked on the UBonn dataset. The classification results on CHB-MIT dataset shows a $94.79 \%$ accuracy with only seven electrodes, as compared to 18 electrodes used in the previous methods [2] [8]. As a result, the proposed work is highly promising, and it needs less computation time than state-ofthe-art work.

\section{CONCLUSION}

In the proposed work, optimized sub-bands of wavelet packet are represented in the phase space for seizures detection on the two data sets UBonn and CHB-MIT. To find optimal sub-bands, mRMR is used on UBonn dataset while optimal number of electrodes were obtained using CHB-MIT data set. The representation of subbands of WPD in the phase space shows promising discriminating ability in the paradigm of seizure detection. The results show that the proposed method provides the classification accuracy much better as compared to the competitive methods at a significantly lower computational cost.

\section{REFERENCES}

[1] Thijs RD, Surges R, O'Brien TJ, Sander JW. "Epilepsy in adults." Lancet. 2019 Feb 16;393(10172):689-701. doi: 10.1016/S01406736(18)32596-0. Epub 2019 Jan 24. PMID: 30686584.

[2] S. Nasiri and G. D. Clifford, "Generalizable Seizure Detection Model Using Generating Transferable Adversarial Features," in IEEE Signal Processing Letters, vol. 28, pp. 568-572, 2021, doi: 10.1109/LSP.2021.3060967. 
[3] Gupta A, Singh P, Karlekar M. "A Novel Signal Modeling Approach for Classification of Seizure and Seizure-Free EEG Signals." IEEE Trans Neural Syst Rehabil Eng. 2018 May;26(5):925-935. doi: 10.1109/TNSRE.2018.2818123. PMID: 29752227.

[4] E. Yavuz, M. C. Kasapba sı, C. Eyüpo glu, and R. Yazıcı, "An epileptic seizure detection system based on cepstral analysis and generalized regression neural network," Biocybern. Biomed.Eng., vol. 38, no. 2, pp. 201-216, 2018.

[5] S. Rukhsar, H. Rehman, Y. U. Khan and A. T. Khan, "Detection of epileptic seizure in EEG signals using phase space reconstruction and euclidean distance of first-order derivative," 2017 International Conference on Inventive Computing and Informatics (ICICI), 2017, pp. 821-826, doi: 10.1109/ICICI.2017.8365251

[6] H. Peng, C. Lei, S. Zheng, C. Zhao, et al., Automatic epileptic seizure detection via Stein kernel-based sparse representation, Comput. Biol. Med. 132 (2021), 104338.

[7] T. Zhang, Z. Han, X. Chen, W. Chen, "Subbands and cumulative sum of subbands based nonlinear features enhance the performance of epileptic seizure detection”, Biomedical Signal Processing and Control, 69(2021), 102827.

[8] A Shankar, H.K Khaing, S. Dandapat, S.Barma, "Analysis of epileptic seizures based on EEG using recurrence plot images and deep learning, Biomedical Signal Processing and Control”, 69(2021), 102854.

[9] Srinivasan, V., Eswaran, C., Sriraam, N. (2007). "Approximate entropybased epileptic EEG detection using artificial neural networks". IEEE Transactions on Information Technology in Biomedicine, 11, 288-295.

[10] V. Gupta, R.B. Pachori, "Epileptic seizure identification using entropy of FBSE based EEG rhythms", Biomed. Signal Process. Control 53 (2019), 101569.

[11] E. Yavuz, M. C. Kasapbaşi, C. Eyupoğlu and R. Yazıcı, "An epileptic seizure detection system based on cepstral analysis and generalized regression neural network", Biocybern. Biomed. Eng., vol. 38, no. 2, pp. 201-216, 2018.

[12] Aayesha, Qureshi, M.B., Afzaal, M. et al. "Machine learning-based EEG signals classification model for epileptic seizure detection". Multimed Tools Appl 80, 17849-17877 (2021). https://doi.org/10.1007/s11042021-10597-6.

[13] A. M. Abdelhameed, H. G. Daoud and M. Bayoumi, "Deep Convolutional Bidirectional LSTM Recurrent Neural Network for Epileptic Seizure Detection," 2018 16th IEEE International New Circuits and Systems Conference (NEWCAS), 2018, pp. 139-143, doi: 10.1109/NEWCAS.2018.8585542.

[14] Abdelhameed A, Bayoumi M (2021) "A deep learning approach for automatic seizure detection in children with epilepsy". Front Comput Neurosci 15:650050. https://doi.org/10.3389/fncom.2021.650050

[15] Z. Zhao, R. Anand and M. Wang, "Maximum Relevance and Minimum Redundancy Feature Selection Methods for a Marketing Machine Learning Platform,” 2019 IEEE International Conference on Data Science and Advanced Analytics (DSAA), 2019, pp. 442-452, doi: 10.1109/DSAA.2019.00059.

[16] C.X. Han ,J. Wang, G.S. Yi,Y.Q. Che, "Investigation of EEG abnormalities in the early stage of Parkinson's disease," Cognitive Neurodynamics, vol. 7, no. 4, pp. 351-359, August, 2017.

[17] Takens F (1981) Detecting strange attractors in turbulence. In:Rand D, Young L-S (eds) Dynamical systems and turbulence, Warwick 1980. Lecture notes in mathematics. Springer, New York, pp 366-381. https://doi.org/10.1007/bfb0091924

[18] A. M. Fraser and H. L. Swinney, "Independent coordinates forstrange attractors from mutual information," Physical Review A, vol. 33, no. 2, pp. 1134-1140, 1986.

[19] M. B. Kennel, R. Brown, and H. D. I. Abarbanel, "Determining embedding dimension for phase-space reconstruction using a geometrical construction," Physical Review A, vol. 45, no. 6, pp. 3403-3411, 1992.

[20] J. A. K. Sukens and J. Vandewalle, "Least squares support vector machine classifiers," Neural Process. Lett., vol. 9, no. 3, pp. 293-300, Jun. 1999

[21] F. Mormann, R. G. Andrzejak, C. E. Elger, and K. Lehnertz, "Seizure prediction: The long and winding road," Brain, vol. 130, no. 2, pp. 314-333, 2007.

[22] A. Hossam Shoeb, "Application of machine learning to epileptic seizure onset detection and treatment," Ph.D. dissertation, HarvardUniv.-MIT Div. Health Sci. Technol., Massachusetts Inst. Technol., Cambridge,MA, USA, 2009. 